\title{
Role of Endoscopic Ultrasound-Guided Fine-Needle Aspiration in the Evaluation of Abdominal Lymphadenopathy of Unknown Etiology
}

\author{
Nonthalee Pausawasdi ${ }^{1,2}$, Kotchakon Maipang ${ }^{1}$, Tassanee Sriprayoon ${ }^{1}$, and Phunchai Charatcharoenwitthaya ${ }^{1,2}$ \\ ${ }^{1}$ Siriraj GI Endoscopy Center, Faculty of Medicine Siriraj Hospital, Mahidol University, Bangkok, ${ }^{2}$ Division of Gastroenterology, \\ Department of Medicine, Faculty of Medicine Siriraj Hospital, Mahidol University, Bangkok, Thailand
}

Background/Aims: Endoscopic ultrasound-guided fine-needle aspiration (EUS-FNA) is a standard procedure for obtaining tissue from lesions near the gastrointestinal lumen. However, there is a scarcity of information on the diagnostic performance of EUS-FNA for abdominal lymphadenopathy of unknown causes. To assess the accuracy of EUS-FNA in diagnosing abdominal lymphadenopathy of unknown etiology.

Methods: The EUS records of patients with undiagnosed abdominal lymphadenopathy between 2010 and 2015 were reviewed.

Results: A total of 42 patients were included in this study. Adequate specimens were obtained from 40 patients (95\%). The final diagnoses were metastatic cancer $(n=16)$, lymphoma $(n=9)$, tuberculosis $(n=8)$, inflammatory changes $(n=6)$, and amyloidosis $(n=1)$. For diagnosing malignancy, EUS-FNA had a sensitivity of $84.6 \%$, specificity of $95.7 \%$, positive predictive value of $91.7 \%$, negative predictive value of $91.7 \%$, and area under the receiver operating characteristic curve (AUROC) of 0.901 . For the diagnosis of lymphoma, EUS-FNA was $100 \%$ accurate when combined with cytologic evaluation and immunohistochemical staining. The diagnostic sensitivity decreased to $75 \%$, whereas the specificity remained $100 \%$, for tuberculosis. The overall AUROC was 0.850 . No procedure-related complications occurred.

Conclusions: EUS-FNA showed high diagnostic performance for abdominal lymphadenopathy of unknown causes, especially malignancy, lymphoma, and tuberculosis. Therefore, it is a crucial diagnostic tool for this patient population. Clin Endosc 2022;55:279-286

Key Words: Endosonography; Fine-needle aspiration; Lymphadenopathy; Lymphoma; Tuberculous lymphadenitis

\section{INTRODUCTION}

Abdominal lymphadenopathy of unknown etiology can be seen in asymptomatic individuals or patients with weight loss, low-grade fever, and abdominal pain. ${ }^{1}$ Establishing a diagnosis can be challenging despite extensive history taking, physical examination, laboratory testing, and cross-sectional imaging. Systemic computed tomography (CT) imaging and

Received: August 12, 2021 Revised: October 16, 2021 Accepted: October 26, 2021

Correspondence: Nonthalee Pausawasdi

Division of Gastroenterology, Department of Internal Medicine, Faculty of Medicine Siriraj Hospital, Mahidol University, Bangkok 10700, Thailand Tel: +662-419-7281, Fax: +662-411-5013, E-mail: nonthaleep7@gmail.com ORCID: https://orcid.org/0000-0002-3737-8555

(c) This is an Open Access article distributed under the terms of the Creative Commons Attribution Non-Commercial License (http://creativecommons.org/ licenses/by-nc/3.0) which permits unrestricted non-commercial use, distribution, and reproduction in any medium, provided the original work is properly cited. various predictive parameters, including the anatomical site and distribution, enhancement patterns, and size of the lymph nodes, have been used to distinguish malignant from benign lymphadenopathies. However, these criteria have limitations, and the normal size limit may vary depending on the location. In addition, most radiologic studies assessing lymph nodes were performed in cohorts with malignant diseases. Normal nodes usually tend to have an oval or a cigar shape and a size of $<1 \mathrm{~cm}$. Generally, rounded nodes $>1 \mathrm{~cm}$ are suggestive of malignancy. Signal intensity changes and dynamic gadolinium contrast enhancement in magnetic resonance imaging (MRI) can help characterize the lymph nodes. ${ }^{2}$ However, imaging alone is not sufficient to provide an accurate diagnosis. ${ }^{3}$ Hence, tissue diagnosis is crucial. Specimens could be obtained using CT-guided biopsy, laparoscopic surgery, or laparotomy. However, the radiologic approach may be limited by the size and location of the lesion. Meanwhile, the invasive nature of surgery limits the utility of this method because the risks may 
outweigh the benefits.

Endoscopic ultrasound-guided fine-needle aspiration (EUS-FNA) offers a means to obtain tissue for the diagnosis of lesions adjacent to the gastrointestinal tract, providing adequate specimen for diagnosis in $>90 \%$ of cases. ${ }^{4-6}$ This tool is essential, especially in isolated abdominal lymphadenopathy, as it can avoid conventional invasive methods. ${ }^{7}$ However, information about the yield of EUS-FNA in this clinical setting is still lacking. The aim of this study was to assess the diagnostic accuracy of EUS-FNA using 22-gauge needles in patients with isolated abdominal lymphadenopathy of unknown etiology.

\section{MATERIALS AND METHODS}

\section{Study population}

The Siriraj Institutional Review Board approved the research protocol, which conformed to the ethical guidelines of the 1975 Helsinki Declaration. The EUS database at a tertiary care center from 2010 to 2015 was retrospectively reviewed. Patients with abdominal lymphadenopathy of unknown etiology who underwent EUS-FNA were identified. The inclusion criteria were as follows: (1) enlarged abdominal lymph nodes detected with MRI or abdominal CT in the absence of an identifiable primary lesion, (2) lymph nodes that can be accessed for EUS-FNA based on abdominal imaging. The exclusion criteria were as follows: (1) superficial lymphadenopathy, (2) known history of malignancy, (3) known history of tuberculosis, (4) definite diagnosis determined using modalities other than EUS-FNA, (5) coagulopathy (international normalized ratio $>1.5$ ), and (6) thrombocytopenia (platelet count $<50,000 \mu \mathrm{L})$.

\section{EUS-FNA technique}

All EUS procedures were performed with a linear echoendoscope (GF-UC140P-AL5; Olympus Optical Co. Ltd., Tokyo, Japan), with the patient under sedation with propofol, by an experienced endosonographer who had performed $>2,000$ EUS procedures. Aspiration was performed using a 22-gauge FNA needle (EZ shot; Olympus Medical, Tokyo, Japan and EchoTip; Cook Medical, IN, USA). The enlarged lymph nodes were assessed under three scanning scope positions, as follows: (1) gastric station, to assess the celiac, peripancreatic (body and tail), and periportal nodes, (2) duodenal bulb station, to evaluate the peripancreatic (head) and periportal nodes, (3) second part of the duodenum, to examine lymph nodes near the uncinate process, mesenteric vessels, and inferior vena cava. Needle puncture was performed through the stomach for position (1) and through the duodenum for positions (2) and (3). The technique involved localizing the target lymph node, Doppler assessment of intervening vessels, needle puncture, tissue aspiration, and specimen collection and processing. Under ultrasound guidance, the lymph node was punctured after the Doppler evaluation confirmed the lack of intervening vessels. The stylet was removed, and negative pressure was applied with $5 \mathrm{~mL}$ of suction, followed by moving the needle to and fro within the lymph node using the fanning technique. The aspirated material was expelled onto glass slides for visual inspection, and the specimen from each pass was prepared for cytology and cell block assessment. If inadequate macroscopic material was observed, repeated passes were performed (up to four passes). Rapid on-site evaluation was not performed.

\section{Cytology and cell block evaluation}

The specimens from each pass were divided for cytology and cell block preparation. The aspirated specimens were expelled on a glass slide, smeared for cytologic assessment, and immediately fixed with $95 \%$ ethyl alcohol solution. The alcohol-fixed slides were stained with Papanicolaou stain for cytologic examination. The remaining material was placed in a bottle containing formalin solution and embedded in paraffin for cell block assessment. The slides were stained with hematoxylin and eosin and reviewed for histologic features. Additional staining, tissue cultures, and immunohistochemical (IHC) studies were performed when necessary.

\section{Data collection}

The demographics, clinical presentations, laboratory results, radiologic findings, EUS findings, cytopathologic results, and clinical course of the study participants were collected. Information on lymph node characteristics on EUS examination, such as size, shape, echogenicity, border, and location, was obtained. The number of needle passes was recorded. The definite diagnosis was determined on the basis of the cytologic or histologic results of EUS-FNA specimens, clinical course, laboratory findings, and results of radiologic follow-up for at least 12 months.

\section{Diagnostic criteria}

The final diagnosis was determined on the basis of the (1) histologic findings of either the surgical specimen or cell blocks, (2) cytologic findings, (3) IHC staining of cell blocks, and (4) clinical course and imaging findings during a minimum follow-up of 12 months. A diagnosis of malignancy was made using the following criteria: (1) surgical histology results suggesting malignancy, (2) cytologic or histologic evidence of malignancy based on EUS-FNA specimens, or (3) cytopathologic results suspicious for malignancy combined with the clinical course and follow-up imaging indicating malignancy. Lymphoma was diagnosed on the basis of histologic findings 
and IHC staining. The diagnosis of tuberculosis was made on the basis of histologic findings of caseating granuloma or positive acid-fast bacilli (AFB) staining of the cell blocks. The diagnosis of other benign conditions required negative cytopathologic assessment and at least 12 months of clinical and imaging follow-up demonstrating no progression or resolution of the enlarged lymph nodes.

\section{Statistical analysis}

The data were analyzed using the Statistical Product and Service Solution (SPSS) statistics software (version 16; SPSS Inc., Chicago, IL, USA). Descriptive statistics were used for demographic, clinical, and laboratory data. Continuous variables were summarized as mean \pm standard deviation, and categorical variables were summarized as percentages. Qualitative variables were summarized as counts and percentages. Comparisons between groups were performed using the $\chi^{2}$ test for categorical variables and the $t$-test for continuous variables. The predictive ability of EUS-FNA was analyzed by calculating the sensitivity, specificity, positive predictive value (PPV), and negative predictive value (NPV) with $95 \%$ confidence intervals (CIs). The area under the receiver operating characteristic curve (AUROC) was estimated to evaluate the overall accuracy of EUS-FNA. Logistic regression analysis was used to determine the factors associated with the presence of malignancy or tuberculosis. The data are presented as odds ratios (ORs) with 95\% CIs. Statistical significance was set at $p<0.05$.

\section{RESULTS}

\section{Characteristics of the study cohort}

A total of 803 patients were identified from the EUS database, with 370 patients undergoing EUS-FNA for intra-abdominal solid mass lesions, during the study period. Of them, 328 patients were excluded because of having solid pancreatic masses, pancreatic cystic lesions, subepithelial lesions, periampullary masses, liver masses, and mediastinal masses. Forty-two patients underwent EUS-FNA for isolated abdominal lymphadenopathy of unknown etiology and fulfilled the eligibility criteria. The baseline characteristics of the patients are shown in Table 1 . The mean age was $52.7 \pm 15.8$ years, and $60 \%$ were men. A vast majority of the patients had at least one symptom, and only five patients (12\%) were asymptomatic. The presenting symptoms included abdominal pain or discomfort (57\%), relevant weight loss (26\%), fever (17\%), anemia (10\%), jaundice (7\%), and a palpable mass (5\%). Most patients (90\%) had multiple lymph nodes.

\section{Etiology of isolated abdominal lymphadenopathy}

Adequate tissue specimens were obtained from 40 patients (95\%), all of whom received a definite diagnosis. The final diagnoses included metastatic cancer $(n=16)$, lymphoma $(n=9)$, tuberculosis $(n=8)$, inflammatory changes $(n=6)$, and amyloidosis $(n=1)$, as shown in Figure 1. Most patients with metastatic cancer had adenocarcinoma of unknown primary site, and the remaining patients had primary pancreatic cancer $(n=3)$, ampullary cancer $(n=1)$, gallbladder cancer $(n=1)$, gastric cancer $(n=1)$, neuroendocrine tumor $(n=1)$, and sarcoma $(n=1)$. Additional IHC staining was performed in 15 cases (35.7\%), with a final diagnosis of lymphoma $(n=9)$, metastatic adenocarcinoma $(n=2)$, neuroendocrine tumor $(n=1)$, sarcoma $(n=1)$, amyloidosis $(n=1)$, and reactive lymphadenopathy $(n=1)$. Among the two patients with inadequate specimens, one patient had positive AFB staining of the aspirated material and was treated for tuberculosis, which reduced the size of the enlarged lymph nodes. The other patient was diagnosed with a benign condition based on the stable clinical course and imaging findings after a 1-year follow-up without progression of the lymphadenopathy. Logistic regression analysis was performed to evaluate the association between clinical parameters and diagnoses. In univariate analysis, age $\geq 55$ years was associated with malignancy (OR, 10.0; 95\% CI, 2.06 to $48.6 ; p=0.002$ ) and fever was associated with tuberculosis (OR, 8.3; 95\% CI, 1.33 to $52.0 ; p=0.013$ ).

Table 1. Baseline Characteristics of the Study Cohort

\begin{tabular}{lc}
\hline Parameters & Value \\
\hline Age (years) & $52.7 \pm 15.8$ \\
Sex & \\
Male & $25(60)$ \\
Female & $17(40)$ \\
Clinical presentation & \\
Abdominal pain & $24(57)$ \\
Weight loss & $11(26)$ \\
Fever & $7(17)$ \\
Asymptomatic & $5(12)$ \\
Anemia & $4(10)$ \\
Jaundice & $3(7)$ \\
Abdominal mass & $2(5)$ \\
\hline
\end{tabular}

Data are presented as mean \pm standard deviation or number (\%). 


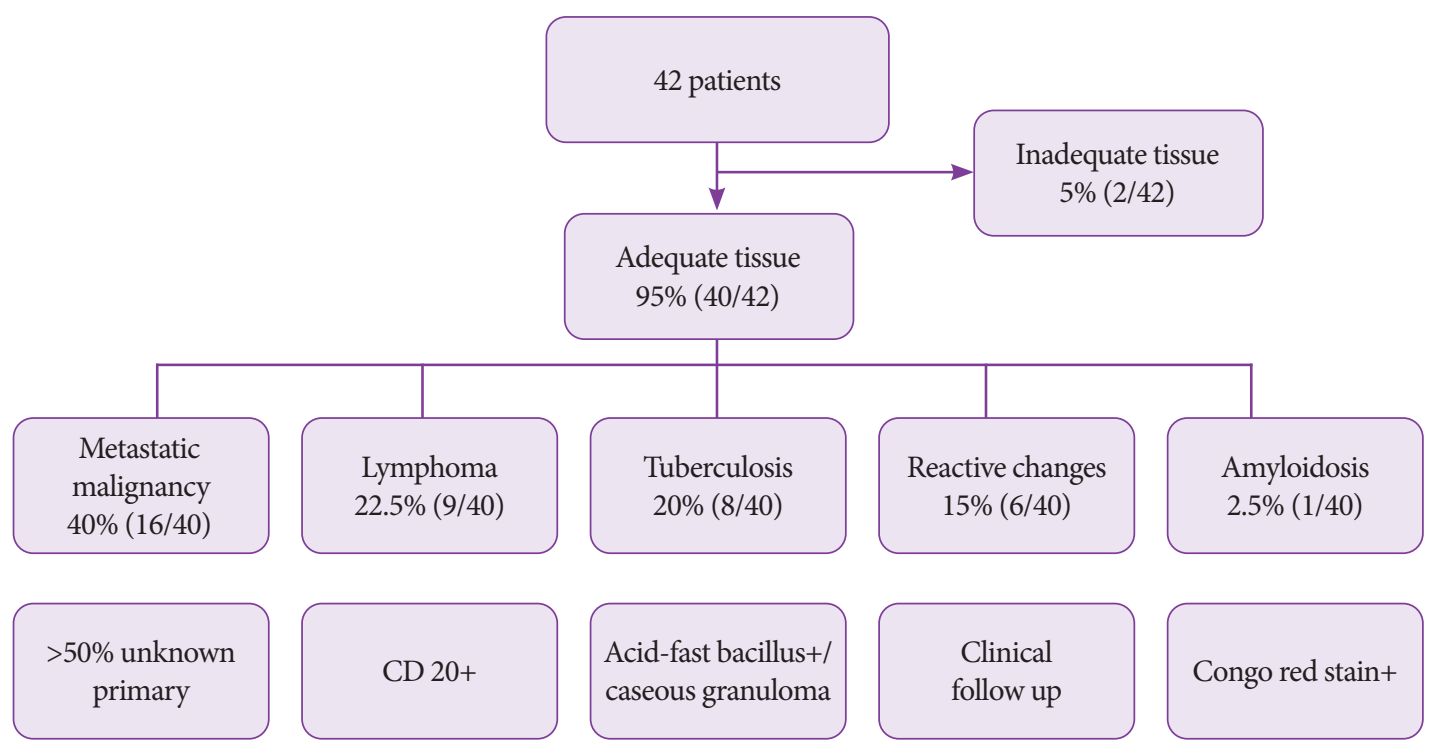

Fig. 1. Final diagnoses of the study cohort.

\section{EUS features of lymph nodes}

The number, location, size, shape, border, and echogenicity of lymph nodes were evaluated. Three patients (7\%) had six nodes. Thirty-five patients ( $83 \%$ ) had to two to five nodes, and four patients $(9.5 \%)$ had a single node. The most common locations of the targeted lymph nodes included the peripancreatic (57\%), celiac (38\%), and aortocaval regions. (24\%). The mean size of the lymph nodes was $24.6 \pm 13.2 \mathrm{~mm}$ (range 5-70 $\mathrm{mm}$ ) in the long axis. Of the visualized lymph nodes, 31 were $>20 \mathrm{~mm}, 15$ were $16-20 \mathrm{~mm}, 14$ were $11-15 \mathrm{~mm}$, and 7 were $<10 \mathrm{~mm}$ in size.

The lymph node shape was described as round, oval, or irregular. With respect to the distribution of the lymph node shape, 24 (42\%) were round, 14 (25\%) were oval, and 18 (32\%) were irregular. The lymph node border was reported as regular or irregular. Twenty-three nodes (53.5\%) had regular borders, whereas 20 (46.5\%) had irregular borders. The echogenic pattern was described as hypoechoic, hyperechoic, or isoechoic Thirty-six lymph nodes (83.7\%) were hypoechogenic, six (13.9\%) were hyperechoic, and one (2.3\%) was isoechoic.

Logistic regression analysis was performed to assess the association between endosonographic features and diagnoses. Univariate analysis indicated that an oval or irregular shape was strongly associated with tuberculosis or inflammatory changes rather than malignancy (OR, 6.0; 95\% CI, 1.08 to $33.3 ; p=0.029$ ). Lymphadenopathy in the perihepatic area was correlated with tuberculosis (OR, 11.0; 95\% CI, 1.75 to 69.1; $p=0.005)$. Moreover, lymph nodes with regular borders were more frequently observed in the lymphoma group than in the non-lymphoma group (OR, 12.6; 95\% CI, 1.35 to 117.6; $p=0.010$ ), as shown in Figure 2. In addition, all patients diagnosed with tuberculosis had pus material in the aspirates, as shown in Figure 3. Although lymph node echogenicity could not predict the diagnosis, the results showed that all lymph nodes observed in the lymphoma group were hypoechoic. The mean number of needle passes was $2.1 \pm 1.5$. No complications occurred.

Furthermore, we evaluated the correlation between EUS and $\mathrm{CT}$ in evaluating the shape of the lymph nodes. We found a $48 \%$ correlation between the two modalities, with irregular shape being the most concordant interpretation.

\section{Diagnostic performance of EUS-FNA}

EUS-FNA showed a respectable overall diagnostic performance for undiagnosed abdominal lymphadenopathy, with an AUROC, sensitivity, specificity, PPV, and NPV of $0.85,86.7 \%$, 83.3\%, 96.3\%, and 55.6\%, respectively, as shown in Table 1 . Moreover, the diagnostic accuracy of EUS-FNA for each diagnosis was assessed. The results showed that EUS-FNA had an excellent accuracy in detecting lymphoma, with a sensitivity and specificity of 100\% (95\% CI, 67.6 to 100) and 100\% (95\% CI, 87.9 to 100), respectively, when combined with cytologic assessment, histologic evaluation of cell blocks, and IHC staining. The diagnostic sensitivity decreased to $75 \%$, whereas the specificity and PPV remained $100 \%$, for tuberculosis. However, the two patients whose histology did not reveal granuloma 


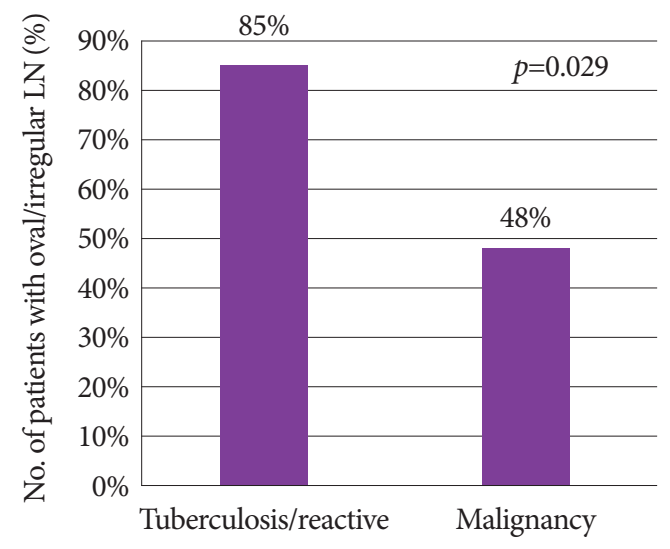

(A)

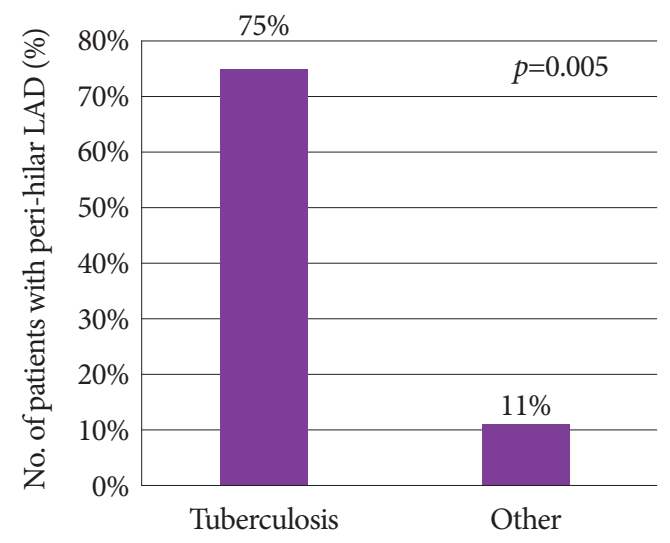

(C)

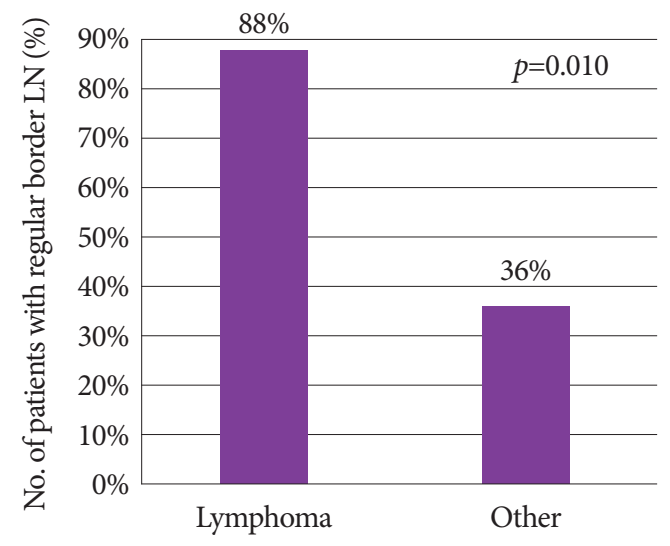

(E)

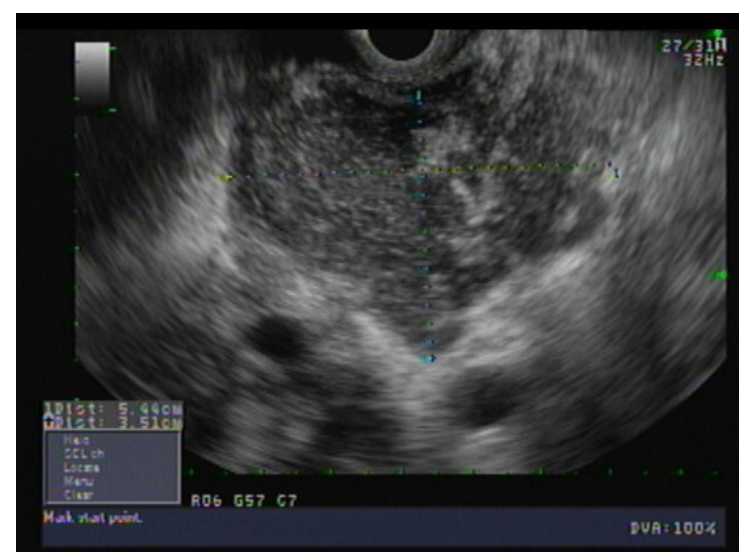

(B)

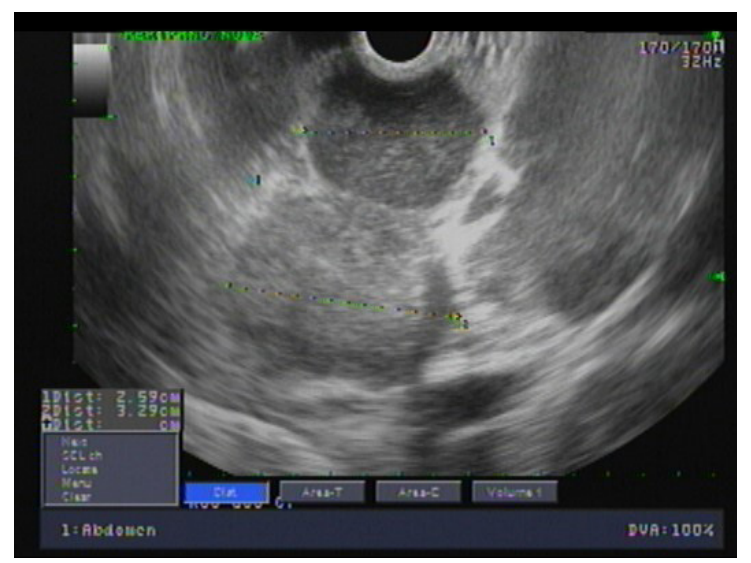

(D)

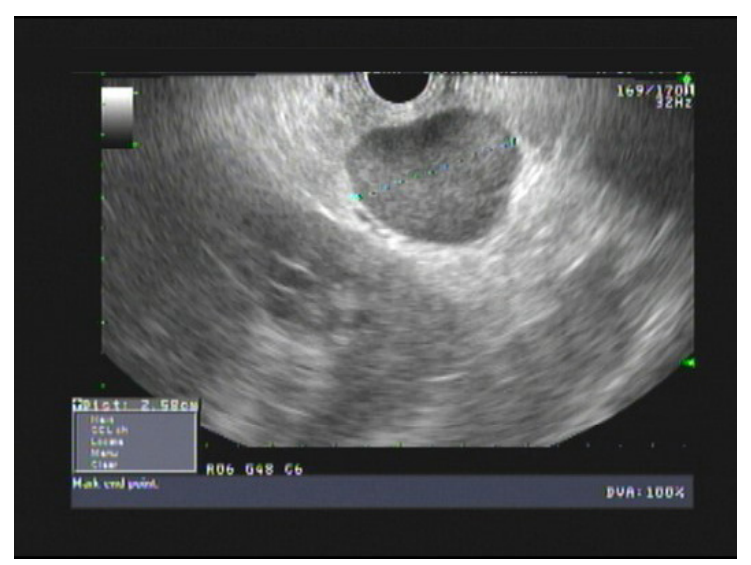

(F)

Fig. 2. The association between endosonographic features and the diagnosis. (A) The bar graph demonstrates the association between the oval or irregular shape of lymph nodes and the diagnosis of tuberculosis or reactive changes compared to malignancy. (B) An endoscopic ultrasound image shows an irregular lymph node. (C) The bar graph demonstrates the association between the peri-hepatic lymph nodes and the diagnosis of tuberculosis. (D) An endoscopic ultrasound image shows enlarged lymph nodes located in the hilar region. (E) The bar graph shows the association between lymph nodes with regular borders and the diagnosis of lymphoma. (F) Endoscopic ultrasound image of a lymph node with a well-defined border. LAD, lymphadenopathy; LN, lymph node. 

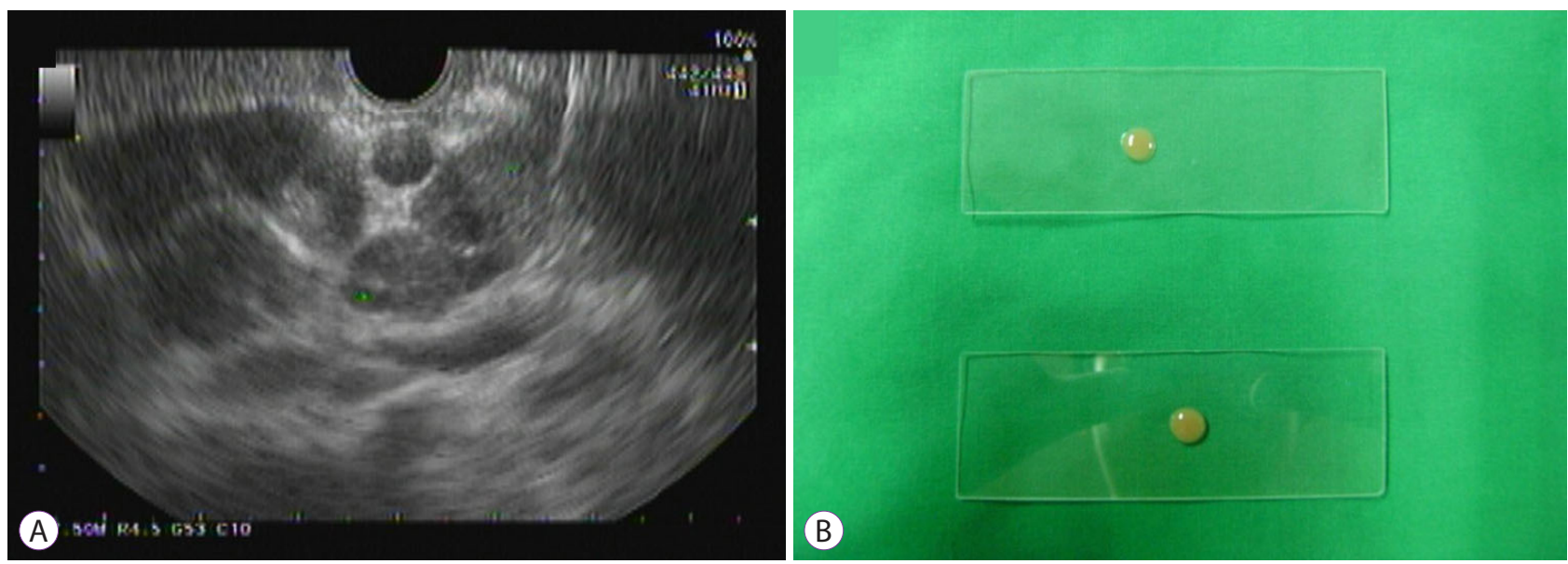

Fig. 3. Characteristic of specimens obtained from a patient with tuberculosis. (A) An endoscopic ultrasound image of a group of peri-hilar lymph nodes in a patient with tuberculosis. (B) Pus in the specimen obtained from fine needle aspiration of a lymph node.

Table 2. Diagnostic Performance of Endoscopic Ultrasound-Guided Fine-Needle Aspiration in the Evaluation of Abdominal Lymphadenopathy

\begin{tabular}{|c|c|c|c|c|c|}
\hline Definite diagnosis of intra-abdominal LAD & AUROC & $\begin{array}{c}\text { Sensitivity } \\
(95 \% \text { CI })\end{array}$ & $\begin{array}{c}\text { Specificity } \\
(95 \% \text { CI })\end{array}$ & $\begin{array}{c}\text { PPV } \\
(95 \% \mathrm{CI})\end{array}$ & $\begin{array}{c}\text { NPV } \\
(95 \% \mathrm{CI})\end{array}$ \\
\hline Overall & 0.850 & $\begin{array}{c}86.7 \\
(70.3-94.7)\end{array}$ & $\begin{array}{c}83.3 \\
(43.6-97.0)\end{array}$ & $\begin{array}{c}96.3 \\
(81.7-99.3)\end{array}$ & $\begin{array}{c}55.6 \\
(26.7-81.1)\end{array}$ \\
\hline Lymphoma & 1.000 & $\begin{array}{c}100 \\
(67.6-100)\end{array}$ & $\begin{array}{c}100 \\
(87.9-100)\end{array}$ & $\begin{array}{c}100 \\
(67.6-100)\end{array}$ & $\begin{array}{c}100 \\
(87.9-100)\end{array}$ \\
\hline Non-hematologic malignancy & 0.901 & $\begin{array}{c}84.6 \\
(57.8-95.7)\end{array}$ & $\begin{array}{c}95.7 \\
(79.0-99.2)\end{array}$ & $\begin{array}{c}91.7 \\
(64.6-98.5)\end{array}$ & $\begin{array}{c}91.7 \\
(74.2-97.7)\end{array}$ \\
\hline Tuberculosis & 0.875 & $\begin{array}{c}75.0 \\
(40.9-92.9)\end{array}$ & $\begin{array}{c}100 \\
(87.9-100)\end{array}$ & $\begin{array}{c}100 \\
(61.0-100)\end{array}$ & $\begin{array}{c}93.3 \\
(78.7-98.2)\end{array}$ \\
\hline
\end{tabular}

AUROC, area under the receiver operating characteristics; CI, confidence interval; LAD, lymphadenopathy; NPV, negative predictive value; PPV, positive predictive value.

were diagnosed with tuberculosis based on positive AFB staining of the aspirates in one patient and positive tissue culture for Mycobacterium tuberculosis in the other patient.

\section{DISCUSSION}

EUS-FNA is a widely accepted method for tissue acquisition in patients with unexplained abdominal lymphadenopathy. This method provides a high diagnostic accuracy of $>90 \%$, without significant complications. ${ }^{1,4,9}$ A previous study suggested that the success rate depends on the number, size, and anatomical accessibility of the enlarged nodes, ${ }^{5}$ with tissue adequacy ranging from $87 \%$ to $95 \%{ }^{1,8,9}$

The differences in the distribution of the etiologies of ab- dominal lymphadenopathy of unknown causes are attributed to the dissimilarity in patient characteristics and geographical variations in disease prevalence. Dhir et al. ${ }^{4}$ reported that tuberculosis was the most common cause $(>50 \%)$ of unexplained intra-abdominal lymphadenopathy in India, a tuberculosis-endemic area, whereas metastatic malignancy and lymphoma accounted for approximately $20 \%$. A Japanese study reported lymphoma as the most common etiology. ${ }^{10}$ Meanwhile, benign diseases (including reactive changes), metastatic malignancy, and lymphoma were the most common findings in Western countries. ${ }^{1,9}$ Although Thailand is a tuberculosis-endemic area, our study revealed that metastatic cancer (40\%) and lymphoma (22.5\%) were the most common causes of unexplained abdominal lymphadenopathy, whereas tuberculosis accounted for $19 \%$. These findings emphasize 
the necessity of EUS-FNA for tissue diagnosis and proper management in this clinical setting. Such discrepancies in disease distribution among tuberculosis-endemic countries are thought provoking. Therefore, we investigated the association between patient characteristics and diagnoses and found a significant association between age $\geq 55$ years and malignancy. In addition, fever was associated with tuberculosis, consistent with the World Health Organization report that fever is one of the most common symptoms of tuberculosis. ${ }^{11}$ The cohort studied by Dhir et al. ${ }^{4}$ was younger (mean age 46.5 years) and had a higher percentage of fever on presentation (59\%) than our cohort (fever was noted in 19\% of our patients).

In the past, endosonographic features were proposed to help differentiate benign from malignant lymph nodes. However, later studies demonstrated the limitations of endosonographic features, and the addition of FNA offered higher diagnostic yield. ${ }^{12}$ In our study, we found some correlations between the diagnoses and endosonographic features (shape, site, and border of lymph nodes). Lymph nodes with an oval or irregular shape were associated with benign diseases, including reactive changes and tuberculosis, rather than malignancy. Perihepatic nodes were associated with tuberculosis. Hypoechoic nodes with well-defined borders were associated with lymphoma. Wang et al..$^{13}$ reported that the patients' sex and age and the location and size of the enlarged nodes were predictors of malignant lymphadenopathy. Celiac location was strongly associated with malignancy, and malignant lymph nodes detected in the celiac axis were more likely to be lymphoma than metastasis. In terms of endosonographic features, metastatic lymph nodes were larger and had an echogenic center, whereas lymphoma-related enlarged lymph nodes had lesion fusion and homogeneous echogenicity. ${ }^{13}$ Therefore, endosonographic findings may provide some diagnostic clues when combined with the clinical spectrum. However, some studies did not find a correlation between endosonographic features and final diagnoses. $^{1,4}$

The success rate of EUS-FNA for adequate sampling was $95 \%$ in our study, consistent with previous studies. ${ }^{1,4,8-10}$ The overall diagnostic accuracy provided an AUROC of 0.850, with a high PPV of $96.3 \%$, emphasizing the low false-positive rate of the test. For the diagnosis of lymphoma, we combined the results of cytologic assessment, histologic evaluation of specimens obtained with a 22 -gauge needle, and additional IHC staining (without flow cytometry owing to the lack of resources). We found that the diagnostic accuracy for lymphoma was $100 \%$, allowing proper treatment and follow-up in all cases. We recognize that lymphoma may not be accurately diagnosed with cytologic examination using conventional FNA, although IHC staining was used in this study. In several lymphoma cases, the diagnosis can be made in certain situa- tions, especially when a monotonous population of lymphoid cells can be obtained for evaluation. For example, if many large lymphoma cells are obtained, IHC staining can provide the definite immunophenotype of lymphoma cells. Even in cases with a predominance of small lymphoid cells with cleaved nuclei that show a BCL2 +CD10+/CD20+/CD3- immunophenotype, the diagnosis of follicular lymphoma can be made with certainty.

The sensitivity slightly decreased for tuberculosis but the specificity and PPV remained excellent. Nonetheless, AFB staining and tissue culture of the aspirates were positive for tuberculosis in patients with a negative cytology, and proper treatment was provided. Furthermore, visualization of pus in the aspirates was indicative of tuberculosis in our cohort. Therefore, purulent specimens should be promptly prepared for AFB staining in this clinical context. The consistency of high diagnostic accuracy and specificity of EUS-FNA (AUROC 0.98 , specificity 98\%) for intra-abdominal lymphadenopathy has been highlighted in a recent meta-analysis including 10 studies with variations in patient demographics and disease prevalence. $^{14}$

The main strengths of this study are that our study population was highly homogeneous and long-term follow-up data were available in all cases. However, some limitations of our study need to be addressed. First, the clinical and EUS features associated with the final diagnoses were derived from only univariate analysis because of sample size limitations in multivariate analysis. Second, surgical pathology was not available in all cases. Lastly, populations with differing prevalence of diseases may limit the generalizability of our results with respect to post-test probabilities; however, the sensitivity and specificity of the tests do not vary across populations.

In summary, EUS-FNA showed high diagnostic performance for abdominal lymphadenopathy of unknown causes. It offered cytopathologic analysis and IHC evaluation for the diagnosis of metastatic cancer, lymphoma, and tuberculosis. Therefore, it is a crucial diagnostic tool for this challenging clinical dilemma.

Conflicts of Interest

The authors have no potential conflicts of interest.

Funding

None.

Acknowledgments

We thank Professor Sanya Sukpanichnant, Department of Pathology, Faculty of Medicine Siriraj Hospital, Mahidol University, Bangkok, Thailand, for reviewing the pathologic slides and providing insightful suggestions on cytologic and pathologic assessments for lymphoma diagnosis. 
Author Contributions

Conceptualization: Nonthalee Pausawasdi

Data curation: Tassanee Sriprayoon

Formal analysis: NP, TS, Phunchai Charatcharoenwitthaya

Investigation: NP

Methodology: NP

Project administration: NP

Supervision: NP

Validation: NP

Visualization: NP

Writing-original draft: NP, Kotchakon Maipang

Writing-review \& editing: NP, KM, PC

\section{ORCID}

Nonthalee Pausawasdi

Kotchakon Maipang

Tassanee Sriprayoon

Phunchai Charatcharoenwitthaya

https://orcid.org/0000-0002-3737-8555

https://orcid.org/0000-0002-3159-103X

https://orcid.org/0000-0001-7046-0529

https://orcid.org/0000-0002-8334-0267

\section{REFERENCES}

1. Korenblit J, Anantharaman A, Loren DE, Kowalski TE, Siddiqui AA. The role of endoscopic ultrasound-guided fine needle aspiration (EUSFNA) for the diagnosis of intra-abdominal lymphadenopathy of unknown origin. J Interv Gastroenterol 2012;2:172-176.

2. Torabi M, Aquino SL, Harisinghani MG. Current concepts in lymph node imaging. J Nucl Med 2004;45:1509-1518.

3. Pongpornsup S, Eksamutchai P, Teerasamit W. Differentiating between abdominal tuberculous lymphadenopathy and lymphoma using multidetector computed tomography (MDCT). J Med Assoc Thai 2013;96:1175-1182.

4. Dhir V, Mathew P, Bhandari S, et al. Endosonography-guided fine needle aspiration cytology of intra-abdominal lymph nodes with unknown primary in a tuberculosis endemic region. J Gastroenterol Hepatol

2011;26:1721-1724

5. Costache M-I, Iordache S, Karstensen JG, Săftoiu A, Vilmann P. Endoscopic ultrasound-guided fine needle aspiration: from the past to the future. Endosc Ultrasound 2013;2:77-85.

6. Wani S, Muthusamy VR, Komanduri S. EUS-guided tissue acquisition: an evidence-based approach (with videos). Gastrointest Endosc 2014;80:939-959.e7.

7. Wiersema MJ, Vilmann P, Giovannini M, Chang KJ, Wiersema LM. Endosonography-guided fine-needle aspiration biopsy: diagnostic accuracy and complication assessment. Gastroenterology 1997;112:10871095.

8. Nakahara O, Yamao K, Bhatia V, et al. Usefulness of endoscopic ultrasound-guided fine needle aspiration (EUS-FNA) for undiagnosed intra-abdominal lymphadenopathy. J Gastroenterol 2009;44:562-567.

9. Coe A, Conway J, Evans J, Goebel M, Mishra G. The yield of EUS-FNA in undiagnosed upper abdominal adenopathy is very high. J Clin Ultrasound 2013;41:210-213.

10. Yasuda I, Tsurumi H, Omar S, et al. Endoscopic ultrasound-guided fine-needle aspiration biopsy for lymphadenopathy of unknown origin. Endoscopy 2006;38:919-924.

11. World Health Organization. Global tuberculosis control : surveillance, planning, financing : WHO report 2007. [Internet]. Geneva: WHO; c2007 [updated 2007; cited 2021 Dec 6]. Available from: http://catalogue. safaids.net/publications/who-report-2007-global-tuberculosis-control-surveillance-planning-financing

12. Chen VK, Eloubeidi MA. Endoscopic ultrasound-guided fine needle aspiration is superior to lymph node echofeatures: a prospective evaluation of mediastinal and peri-intestinal lymphadenopathy. Am J Gastroenterol 2004;99:628-633.

13. Wang J, Chen Q, Wu X, Wang Y, Hou W, Cheng B. Role of endoscopic ultrasound-guided fine-needle aspiration in evaluating mediastinal and intra-abdominal lymphadenopathies of unknown origin. Oncol Lett 2018;15:6991-6999.

14. Li C, Shuai Y, Zhou X. Endoscopic ultrasound guided fine needle aspiration for the diagnosis of intra-abdominal lymphadenopathy: a systematic review and meta-analysis. Scand J Gastroenterol 2020;55:114-122. 